\title{
IMAGING USING TRANSPORT MODELS FOR WAVE-WAVE CORRELATIONS
}

\author{
GUILLAUME BAL * ${ }^{*}$ OLIVIER PINAUD ${ }^{\dagger}$
}

June 8, 2010

\begin{abstract}
We consider the imaging of objects buried in unknown heterogeneous media. The medium is probed by using classical (e.g., acoustic or electromagnetic) waves. When heterogeneities in the medium become too strong, inversion methodologies based on a microscopic description of wave propagation (e.g., a wave equation or Maxwell's equations) become strongly dependent on the unknown details of the heterogeneous medium. In some situations, it is preferable to use a macroscopic model for a quantity that is quadratic in the wave fields. Here, such macroscopic models take the form of radiative transfer equations also referred to as transport equations. They can model either the energy density of the propagating wave fields or more generally the correlation of two wave fields propagating in possibly different media. In particular, we consider the correlation of the two fields propagating in the heterogeneous medium when the inclusion is absent and present, respectively. We present theoretical and numerical results showing that reconstructions based on this correlation are more accurate than reconstructions based on measurements of the energy density.
\end{abstract}

\section{Random media; Imaging; Radiative transfer \\ AMS Subject Classification: 22E46, 53C35, 57S20}

\section{Introduction}

The problem of imaging in complex (highly heterogeneous) environments arises in many important applications such as biomedical imaging, seismic exploration in geophysics [19] and non-destructive testing of materials [29]. One standard imaging technique consists in probing a medium by sending acoustic, electromagnetic, or elastic waves depending on the applications from a localized source and by collecting the echos on an array of detectors.

\footnotetext{
*Department of Applied Physics and Applied Mathematics, Columbia University, New York NY, 10027; gb2030@columbia.edu;

†Université de Lyon, Université Lyon 1, CNRS, UMR 5208 Institut Camille Jordan/ISTIL, Bâtiment du Doyen Jean Braconnier, 43, blvd du 11 novembre 1918, F - 69622 Villeurbanne Cedex, France; pinaud@math.univ-lyon1.fr
} 
Reconstructions then depend on whether the heterogeneous medium is known a priori or not. In the somewhat unlikely event that it is, several classical techniques are available such as e.g. time reversal methods which perform well for the localization of buried inclusions [20]: in the context of acoustic waves, the measured field at the detector is time-reversed, which consists in changing the sign of the velocity and sending back first the signal that has been recorded last, and will peak at the location of the most diffractive inclusion. Back-propagation is possible in this configuration because the medium is known.

When it is not, the imaging problem is different. The unknown medium of propagation may then be modeled as a particular realization of a random medium. The average (slow) part of the medium may be assumed to be known while the local fluctuations are rapid and unknown. The imaging method then depends on the strength of the random heterogeneities and on the relation between the wavelength and the scale at which the medium varies, i.e., the correlation length of the random medium.

When the strength of the heterogeneities is weak, interferometry methods are efficient $[14,15,16,17]$ : since the fluctuations are weak, the wave is only slightly scattered by the medium, so that coherent information is available. Such information propagating in the homogeneous (average) known medium, the technique consists in back-propagating available measurements in the homogeneous medium to observe a peak at the inclusion location.

When the fluctuations are strong, scattering can no longer be neglected and thus needs to be modeled. Scattering is mostly generated by heterogeneities at the same scale as the propagating waves. We thus consider the situation where the wavelength and the correlation length in the random medium are comparable. In such a regime, the dynamics of the wave energy is well described by radiative transfer equations, whose constitutive parameters depend on the statistics of the random medium [18, 28, 23, 27]. The presence of an inclusion is then seen as a perturbation of such parameters. The imaging problem thus consists in solving an inverse transport problem. The major limitation of transport models is their statistical instability. Transport equations are deterministic and model the ensemble average of the energy density. Available measurements typically correspond to one realization of the random medium and thus are not averaged over realizations. It turns out that self-averaging effects allow us to show that the energy density of the waves becomes asymptotically independent of the realization of the random medium as the wavelength tends to zero; see e.g. [6, 10, 26]. This should be seen as an application of the law of large numbers. Numerical simulations $[8,9,11]$ of acoustics waves also demonstrate that measurements over sufficiently large detectors are indeed rather stable statistically, i.e., have small statistical variance.

This paper considers time-dependent acoustic waves. We refer the reader to [11] for similar results in the case of time-harmonic waves. The imaging problem is solved by using transport models introduced e.g. in [9]. Such models describe the propagation of the energy density of the wave as well as the correlation of the wave fields in the presence and in the absence of an inclusion. Constructing such a correlation assumes that both fields (with and without the inclusion) can be measured while energy measurements only require measurements in the presence of the inclusion.

The objectives of the paper are as follows: (i) to demonstrate the imaging capabilities of the transport models, (ii) to assess which observables between the energy and 
the correlation should preferably be used and in which situation, (iii) to quantify the sensitivity of the reconstructions with respect to the statistical instabilities.

Even though perfect statistical stability (vanishing variance) holds in the limit of vanishing wavelength, some instabilities remain in practice since the wavelength is small but finite. Quantifying how much the reconstructions depend on the medium fluctuations is important for the applicability of the method. We will see that correlation measurements exhibit more stable reconstructions in general than energy measurements.

The paper is structured as follows: in section 2, we present the transport equations used to model the wave energy density and field-field correlations. In section 3, we detail the imaging procedure and use formal arguments to show that correlations are less sensitive to the statistical instabilities than energies in the transport regime. We present in section 4 several numerical simulations validating the theory. We reconstruct the transport parameters of the background medium and use radiative transfer equations to image buried inclusions from wave measurements. In particular, we take advantage in one configuration of the strong interaction between the wave and the medium to image an inclusion hidden behind a blocker with no line of sight.

\section{The models}

In this section, we recall the transport equations verified by the wave energy densities and field-field correlations in the high frequency regime.

\subsection{High frequency waves}

The propagation of acoustic waves is described by the following scalar wave equation for the pressure $p$ :

$$
\frac{\partial^{2} p}{\partial t^{2}}=\kappa^{-1}(\mathbf{x}) \nabla \cdot \rho^{-1}(\mathbf{x}) \nabla p, \quad \mathbf{x} \in \mathbb{R}^{d}, t>0
$$

supplemented with initial conditions $p(t=0, \mathbf{x})$ and $\frac{\partial p}{\partial t}(t=0, \mathbf{x})$. Here, $d$ is dimension and $\rho$ and $\kappa$ are density and compressibility of the underlying medium, respectively. For simplicity, we assume that $\rho(\mathbf{x}):=\rho_{0}$ is constant. Generalizations to non-constant densities are possible. The compressibility $\kappa$ is of the form

$$
\kappa(\mathbf{x})=\kappa_{0}(\mathbf{x})+\sigma_{0} \kappa_{1}\left(\frac{\mathbf{x}}{l_{c}}\right)
$$

where $\kappa_{0}$ is the background compressibility (supposed to be known and smooth) and $\kappa_{1}$ accounts for random fluctuations of strength $\sigma_{0}$ and correlation length $l_{c}$. $\kappa_{1}$ is a mean zero, homogeneous stationary random field with correlation function

$$
\mathbb{E}\left\{\kappa_{1}(\mathbf{x}) \kappa_{1}(\mathbf{y})\right\}=R(\mathbf{x}-\mathbf{y}) .
$$

Above, $\mathbb{E}\{\cdot\}$ denotes ensemble average over the different realizations of the random medium. $R$ is assumed to decay fast enough so as to avoid long-range interaction 
effects. We suppose the initial condition has slow and rapid variations (corresponding to a typical length $\lambda$, e.g. a wavelength or a support size) so that

$$
p(t=0, \mathbf{x})=p_{0}\left(\mathbf{x}, \frac{\mathbf{x}}{\lambda}\right), \quad \frac{\partial p}{\partial t}(t=0, \mathbf{x})=p_{1}\left(\mathbf{x}, \frac{\mathbf{x}}{\lambda}\right) .
$$

We consider initial conditions oscillating at the frequency $\lambda^{-1} \gg 1$ with support of order one compared to $\lambda$.

In the regime of weak coupling $[18,28]$, it is assumed that the strength of the fluctuations $\sigma_{0}$ is weak and that the correlation length of the medium $l_{c}$ and the typical length of the initial condition $\lambda$ are same order. Considering stronger fluctuations would possibly yield a stochastic behavior of the wave energy in the high frequency limit, see [21] for instance. This is not the regime we are interested in this paper since one of our objectives is to quantity the statistical stability of the reconstructions, that is how much some reconstructed parameters depend on the realization of the random medium. The stochastic homogeneization case $\lambda \gg l_{c}$ leads to waves propagating in an effective medium, see [3], while the case $\lambda \ll l_{c}$ leads to random Liouville equations [4]. Following the above discussion, we then set

$$
l_{c}=\lambda=\sigma_{0}^{2}=\varepsilon \ll 1 .
$$

The fact that $\sigma_{0}=\sqrt{\varepsilon}$ insures the random medium has an effect of order $\mathcal{O}(1)$ at the macroscopic level. We rewrite (1) to obtain the following high frequency wave equation:

$$
\frac{\partial^{2} p^{\varepsilon}}{\partial t^{2}}=c_{\varepsilon}^{2}(\mathbf{x}) \Delta p^{\varepsilon}, \quad p^{\varepsilon}(t=0, \mathbf{x})=p_{0}\left(\mathbf{x}, \frac{\mathbf{x}}{\varepsilon}\right), \quad \frac{\partial p^{\varepsilon}}{\partial t}(t=0, \mathbf{x})=p_{1}\left(\mathbf{x}, \frac{\mathbf{x}}{\varepsilon}\right)
$$

where $c_{\varepsilon}(\mathbf{x})=\left(\rho_{0} \kappa^{\varepsilon}(\mathbf{x})\right)^{-1 / 2}$ with

$$
\kappa^{\varepsilon}(\mathbf{x})=\kappa_{0}(\mathbf{x})+\sqrt{\varepsilon} \kappa_{1}\left(\frac{\mathbf{x}}{\varepsilon}\right) .
$$

The asymptotic analysis of (2) as $\varepsilon \rightarrow 0$ is done by means of Wigner transforms (see section 2.2 and [27]). We recast the scalar wave equation as a first-order hyperbolic system (see [2] for the high frequency limit from the second order equation) and introduce the acoustic field $\mathbf{u}^{\varepsilon}=\left(\mathbf{v}^{\varepsilon}, p^{\varepsilon}\right)$, where $\mathbf{v}^{\varepsilon}$ is velocity and obtain the following system:

$$
\rho_{0} \frac{\partial \mathbf{v}^{\varepsilon}}{\partial t}+\nabla p^{\varepsilon}=0, \quad \kappa^{\varepsilon} \frac{\partial p^{\varepsilon}}{\partial t}+\nabla \cdot \mathbf{v}^{\varepsilon}=0
$$

augmented with initial conditions $p^{\varepsilon}(t=0, \mathbf{x})$ and $\mathbf{v}^{\varepsilon}(t=0, \mathbf{x})=\nabla \varphi^{\varepsilon}(\mathbf{x})$ where the pressure potential $\varphi^{\varepsilon}$ is obtained by solving

$$
\Delta \varphi^{\varepsilon}=-\kappa_{0} \frac{\partial p^{\varepsilon}}{\partial t}(t=0, \cdot)
$$

The propagation of high frequency waves is thus described by the latter system in the absence of inclusions. We now take into account the presence of inclusions. 
Modeling of the inclusion. We consider two fields: $\mathbf{u}_{1}^{\varepsilon}$, which corresponds to waves propagating in a medium without inclusion, and $\mathbf{u}_{2}^{\varepsilon}$ corresponding to waves propagating a medium with an inclusion. We denote by $\Omega$ the domain of the inclusion. Assuming there are no random fluctuations at its location, the inclusion is typically modeled as a jump of the constitutive parameters of the medium, namely the density and compressibility. Here, we simplify the analysis by assuming that the inclusion is a sound soft inclusion, which is equivalent to setting an infinite sound speed within the inclusion. This leads to vanishing Dirichlet conditions for the pressure $p^{\varepsilon}$ on $\partial \Omega$. We thus obtain the following two systems for the fields $\mathbf{u}_{i}^{\varepsilon}=\left(\mathbf{v}_{i}^{\varepsilon}, p_{i}^{\varepsilon}\right), i=1,2$ :

$$
\begin{array}{llll}
\rho_{0} \frac{\partial \mathbf{v}_{1}^{\varepsilon}}{\partial t}+\nabla p_{1}^{\varepsilon}=0, & \kappa^{\varepsilon} \frac{\partial p_{1}^{\varepsilon}}{\partial t}+\nabla \cdot \mathbf{v}_{1}^{\varepsilon}=0, & \text { in } \mathbb{R}^{d}, \\
\rho_{0} \frac{\partial \mathbf{v}_{2}^{\varepsilon}}{\partial t}+\nabla p_{2}^{\varepsilon}=0, & \kappa^{\varepsilon} \frac{\partial p_{2}^{\varepsilon}}{\partial t}+\nabla \cdot \mathbf{v}_{2}^{\varepsilon}=0, & \text { in } \mathbb{R}^{d} \backslash \bar{\Omega}, & p_{2}^{\varepsilon}=0,
\end{array}
$$

\subsection{Transport equations}

The high frequency limit may be obtained by using Wigner transforms [22, 24], which provides a phase space description of the propagation of the wave energy. The wave energy satisfies transport equations whose constitutive parameters are deduced from the microscopic sound speed $c_{\varepsilon}$ [27]. For the two fields $\mathbf{u}_{i}^{\varepsilon}$, the Wigner transform is defined as the following matrix-valued function:

$$
W^{\varepsilon}\left[\mathbf{u}_{i}^{\varepsilon}, \mathbf{u}_{j}^{\varepsilon}\right](t, \mathbf{x}, \mathbf{k})=\frac{1}{(2 \pi)^{d}} \int_{\mathbb{R}^{d}} e^{i \mathbf{k} \cdot \mathbf{y}} \mathbf{u}_{i}^{\varepsilon}\left(t, \mathbf{x}-\frac{\varepsilon \mathbf{y}}{2}\right) \otimes \mathbf{u}_{j}^{\varepsilon}\left(t, \mathbf{x}+\frac{\varepsilon \mathbf{y}}{2}\right) d \mathbf{y} .
$$

We introduce the notations $W_{1}^{\varepsilon}:=W^{\varepsilon}\left[\mathbf{u}_{1}^{\varepsilon}, \mathbf{u}_{1}^{\varepsilon}\right], W_{2}^{\varepsilon}:=W^{\varepsilon}\left[\mathbf{u}_{2}^{\varepsilon}, \mathbf{u}_{2}^{\varepsilon}\right]$ and $W_{12}^{\varepsilon}:=W^{\varepsilon}\left[\mathbf{u}_{1}^{\varepsilon}, \mathbf{u}_{2}^{\varepsilon}\right]$. Let $\mathcal{E}^{\varepsilon}$ be the energy in absence of inclusion, $\mathcal{E}_{\text {inc }}^{\varepsilon}$ be the energy in presence of inclusion and let $\mathcal{C}^{\varepsilon}$ be the correlation of the two fields $\mathbf{u}_{1}^{\varepsilon}$ and $\mathbf{u}_{2}^{\varepsilon}$. They are respectively defined as

$$
\begin{aligned}
\mathcal{E}^{\varepsilon}(t, \mathbf{x}) & :=\frac{1}{2}\left(\kappa^{\varepsilon}(\mathbf{x})\left(p_{1}^{\varepsilon}(t, \mathbf{x})\right)^{2}+\rho_{0}\left|\mathbf{v}_{1}^{\varepsilon}(t, \mathbf{x})\right|^{2}\right) \\
\mathcal{E}_{\text {inc }}^{\varepsilon}(t, \mathbf{x}) & :=\frac{1}{2}\left(\kappa^{\varepsilon}(\mathbf{x})\left(p_{2}^{\varepsilon}(t, \mathbf{x})\right)^{2}+\rho_{0}\left|\mathbf{v}_{2}^{\varepsilon}(t, \mathbf{x})\right|^{2}\right), \\
\mathcal{C}^{\varepsilon}(t, \mathbf{x}) & :=\frac{1}{2}\left(\kappa^{\varepsilon}(\mathbf{x}) p_{1}^{\varepsilon}(t, \mathbf{x}) p_{2}^{\varepsilon}(t, \mathbf{x})+\rho_{0} \mathbf{v}_{1}^{\varepsilon}(t, \mathbf{x}) \cdot \mathbf{v}_{2}^{\varepsilon}(t, \mathbf{x})\right)
\end{aligned}
$$

It is shown in [27] that in the limit $\varepsilon \rightarrow 0$, the expectation of the Wigner transform $\mathbb{E}\left\{W_{1}^{\varepsilon}\right\}$ tends to a measure $W_{1}$ admitting the following decomposition:

$$
W_{1}(t, \mathbf{x}, \mathbf{k})=a_{1}^{+}(t, \mathbf{x}, \mathbf{k}) \mathbf{b}^{+}(\mathbf{x}, \mathbf{k}) \otimes \mathbf{b}^{+}(\mathbf{x}, \mathbf{k})+a_{1}^{-}(t, \mathbf{x}, \mathbf{k}) \mathbf{b}^{-}(\mathbf{x}, \mathbf{k}) \otimes \mathbf{b}^{-}(\mathbf{x}, \mathbf{k}),
$$

where we have defined

$$
\mathbf{b}^{ \pm}(\mathbf{x}, \mathbf{k})=\frac{1}{\sqrt{2 \rho_{0}}}\left(\begin{array}{c}
\hat{\mathbf{k}} \\
\pm \rho_{0} c_{0}^{-1}(\mathbf{x})
\end{array}\right), \quad c_{0}(\mathbf{x}):=\left(\rho_{0} \kappa_{0}(\mathbf{x})\right)^{-1 / 2}, \quad \hat{\mathbf{k}}:=\frac{\mathbf{k}}{|\mathbf{k}|} .
$$

The absence of vortical modes in the latter expression follows from the form of initial condition $\mathbf{u}_{1}^{\varepsilon}(t=0, \cdot)=\left(\nabla \varphi^{\varepsilon}, p^{\varepsilon}(t=0, \cdot)\right)$. In the absence of an inclusion and when the 
average velocity $c_{0}$ is independent of position, the amplitude of the propagating mode $a_{1}^{+}$solves the following radiative transfer equation:

$$
\frac{\partial a_{1}^{+}}{\partial t}+c_{0} \hat{\mathbf{k}} \cdot \nabla_{\mathbf{x}} a_{1}^{+}+\Sigma(\mathbf{k}) a_{1}^{+}=Q\left(a_{1}^{+}\right), \quad \mathbf{x} \in \mathbb{R}^{d}
$$

augmented with prescribed initial conditions $a_{1}^{+}(0, \mathbf{x}, \mathbf{k})$, where $Q$ and $\Sigma^{-1}$ denote the collision operator and mean free time, respectively, and are given by:

$$
Q(a)=\int_{\mathbb{R}^{d}} a(t, \mathbf{x}, \mathbf{p}) \sigma(\mathbf{k}, \mathbf{p}) \delta\left(c_{0}|\mathbf{p}|-c_{0}|\mathbf{k}|\right) d \mathbf{p}, \quad \Sigma(\mathbf{k})=\int_{\mathbb{R}^{d}} \sigma(\mathbf{k}, \mathbf{p}) \delta\left(c_{0}|\mathbf{p}|-c_{0}|\mathbf{k}|\right) d \mathbf{p} .
$$

The cross section $\sigma(\mathbf{k}, \mathbf{p})$ appearing in these expressions is given by

$$
\sigma(\mathbf{k}, \mathbf{p})=\frac{\pi c_{0}^{2}|\mathbf{k}|^{2}}{2(2 \pi)^{d}} \hat{R}(\mathbf{k}-\mathbf{p})
$$

A similar expression is obtained for $a_{1}^{-}(t, \mathbf{x}, \mathbf{k})=a_{1}^{+}(t, \mathbf{x},-\mathbf{k})$. The expectation of the physical energy density is then derived from the phase-space energy density by averaging out the $\mathbf{k}$ variable:

$$
\lim _{\varepsilon \rightarrow 0} \mathbb{E}\left\{\mathcal{E}^{\varepsilon}\right\}(t, \mathbf{x})=\int_{\mathbb{R}^{d}} a_{1}^{+}(t, \mathbf{x}, \mathbf{k}) d \mathbf{k} .
$$

The question of the statistical stability of the limit, that is determining whether the random variable $W_{1}^{\varepsilon}$ converges to its average $\mathbb{E}\left\{W_{1}^{\varepsilon}\right\}$ (and therefore to $W_{1}$ ) in the high frequency limit, is addressed in section 2.3. Regarding $W_{2}^{\varepsilon}$ and $W_{12}^{\varepsilon}$, we have for $\mathbf{x} \in$ $\mathbb{R}^{d} \backslash \bar{\Omega}$

$$
\begin{aligned}
W_{2}(t, \mathbf{x}, \mathbf{k}) & =a_{2}^{+}(t, \mathbf{x}, \mathbf{k}) \mathbf{b}^{+}(\mathbf{x}, \mathbf{k}) \otimes \mathbf{b}^{+}(\mathbf{x}, \mathbf{k})+a_{2}^{-}(t, \mathbf{x}, \mathbf{k}) \mathbf{b}^{-}(\mathbf{x}, \mathbf{k}) \otimes \mathbf{b}^{-}(\mathbf{x}, \mathbf{k}) \\
W_{12}(t, \mathbf{x}, \mathbf{k}) & =a_{12}^{+}(t, \mathbf{x}, \mathbf{k}) \mathbf{b}^{+}(\mathbf{x}, \mathbf{k}) \otimes \mathbf{b}^{+}(\mathbf{x}, \mathbf{k})+a_{12}^{-}(t, \mathbf{x}, \mathbf{k}) \mathbf{b}^{-}(\mathbf{x}, \mathbf{k}) \otimes \mathbf{b}^{-}(\mathbf{x}, \mathbf{k})
\end{aligned}
$$

where $a_{2}^{ \pm}$and $a_{12}^{ \pm}$satisfy the transport equation (4) in $\mathbb{R}^{d} \backslash \bar{\Omega}$. At the inclusion's boundary $\partial \Omega$, the energy is specularly reflected since the inclusion is non-penetrable, and $a_{2}^{ \pm}$ therefore satisfies specular boundary conditions on $\partial \Omega$. Regarding the correlation $\mathcal{C}^{\varepsilon}$, it is shown in [9] that it must verify vanishing Dirichlet conditions on $\partial \Omega$ since the two wave fields $\mathbf{u}_{1}^{\varepsilon}$ and $\mathbf{u}_{2}^{\varepsilon}$ satisfy different dispersion relations inside the inclusion. We thus prescribe the following boundary conditions for the energy and correlation amplitudes $a_{2}^{+}$and $a_{12}^{+}$:

$$
\begin{aligned}
a_{2}^{+}(t, \mathbf{x}, \mathbf{k}) & =a_{2}^{+}(t, \mathbf{x}, \mathbf{k}-2 \mathbf{k} \cdot \mathbf{n}(\mathbf{x}) \mathbf{n}(\mathbf{x})), \quad \mathbf{x} \in \partial \Omega \\
a_{12}^{+}(t, \mathbf{x}, \mathbf{k}) & =0, \quad \mathbf{x} \in \partial \Omega, \mathbf{k} \cdot \mathbf{n}(\mathbf{x})>0
\end{aligned}
$$

Above, $\mathbf{n}(\mathbf{x})$ is the outward normal to the boundary $\partial \Omega$ at $\mathbf{x} \in \partial \Omega$. In the same manner as (7), the physical energy density in presence of inclusion and the correlation of the two fields are obtained by averaging out the $\mathbf{k}$ variable:

$$
\lim _{\varepsilon \rightarrow 0} \mathbb{E}\left\{\mathcal{E}_{\text {inc }}^{\varepsilon}\right\}(t, \mathbf{x})=\int_{\mathbb{R}^{d}} a_{2}^{+}(t, \mathbf{x}, \mathbf{k}) d \mathbf{k}, \quad \lim _{\varepsilon \rightarrow 0} \mathbb{E}\left\{\mathcal{C}^{\varepsilon}\right\}(t, \mathbf{x})=\int_{\mathbb{R}^{d}} a_{12}^{+}(t, \mathbf{x}, \mathbf{k}) d \mathbf{k} .
$$

To summarize, the expectation of waves energies with and without an inclusion, $\mathcal{E}^{\varepsilon}$ and $\mathcal{E}_{\text {inc }}^{\varepsilon}$, as well as the expectation of correlation $\mathcal{C}^{\varepsilon}$, can be described in the high frequency 
limit by the amplitudes $a_{1}^{+}, a_{2}^{+}$and $a_{12}^{+}$, respectively, satisfying the transport equation (4) with specular and homogeneous Dirichlet boundary conditions at the inclusion's boundary for the last two amplitudes. The transport equation depends only on the fluctuations of the random medium through its power spectrum $\hat{R}$.

It remains to understand how close $\mathcal{E}^{\varepsilon}$ is to $\mathbb{E}\left\{\mathcal{E}^{\varepsilon}\right\}$ and how the limits in (7) and (8) should be interpreted. This the object of the next section.

\subsection{Statistical stability}

Statistical stability is an essential property required for transport-based imaging. Indeed, from waves measurements of the energies $\mathcal{E}^{\varepsilon}$ and $\mathcal{E}_{\text {inc }}^{\varepsilon}$, as well as the correlation $\mathcal{C}^{\varepsilon}$, we intend to perform reconstructions using the different transport equations on the amplitudes $a_{i}^{+}, i=1,2,12$. These amplitudes accurately describe the expectation of the macroscopic energies within the high frequency limit. In practical experiments, these expectations are not available since measurements are performed for only one medium, namely the physical medium of interest. The question is thus whether the deterministic limiting amplitudes correctly model the behavior of the random variables $\mathcal{E}^{\varepsilon}, \mathcal{E}_{\text {inc }}^{\varepsilon}$, and $\mathcal{C}^{\varepsilon}$

Some mathematical results address this problem within the paraxial approximation in which the wave equation is replaced by a random Schrödinger equation: under different assumptions on the random medium, it is proved in $[1,6,10]$ for instance that the Wigner transform of the random wavefunction converges weakly and in probability to a measure solution to the transport equation. This implies the statistical stability of wave energy in the paraxial regime for some types of random fluctuations, so that the transport equations indeed accurately describe the propagation of the (random) energy in the high frequency limit. The energy needs to be spatially averaged over a sufficiently large domain since the convergence is meant in a weak sense. It is shown in addition in [10] that the stability is related to the regularity of the initial conditions. The question of statistical stability for the standard wave equation remains an open problem. For discrete waves, it is proved in [25] that the expectation of the wave energy can be modeled by transport equations in the high frequency limit.

Extrapolating on the mathematical results obtained for the paraxial regime, it is likely that the statistical stability also holds for the wave equation, that is $\mathbb{E}\left\{\mathcal{E}^{\varepsilon}\right\} \approx \mathcal{E}^{\varepsilon}$ as $\varepsilon$ goes to zero. That property has been verified numerically for a small but non-zero value of $\varepsilon$ in various two dimensional settings in $[7,8,9]$, and we also present some simulations in section 4 that are consistent with statistical stability.

\section{Imaging the inclusion}

We present here the procedure we use to reconstruct the inclusion. The main parameter characterizing the random medium is the strength of the fluctuations, which can be measured by their standard deviation $R(\mathbf{0})$. Directly related to the strength of the fluctuations is the mean free path $l:=c_{0} \Sigma^{-1}$. When the standard deviation is small, waves propagating are not too affected by the fluctuations so that we have access to coherent information, for instance the wave front which is only slightly distorted by the propagation in the random medium. In such a configuration, interferometry methods, 
which consist in backpropagating the data in a homogeneous medium to observe a recompression at the inclusion location, perform well as shown e.g. in [14].

In this paper, we are interested in stronger fluctuations with significantly smaller mean free paths. In this setting, available data are primarily incoherent, that is the fraction of the wave that has been scattered by the random medium is dominant. The coherent part of the waves is damped by the fluctuations and becomes negligible compared to the incoherent part. Imaging in this configuration is more difficult and is possible essentially in two cases, see $[9,11]$; (i) either the inclusion is large enough to overcome statistical instabilities and reconstructions can be performed from knowledge of the energy in presence of inclusion $\mathcal{E}_{\text {inc }}^{\varepsilon}$; or (ii) the inclusion's influence on the data is smaller and in this case differential measurements are needed, that is we need to measure the energies both in the presence and in the absence of the inclusion. In the latter case, the measurements are $\mathcal{E}_{\text {inc }}^{\varepsilon}-\mathcal{E}^{\varepsilon}$ and the statistical instabilities are reduced since they are now proportional to the size of the inclusion [9]. Indeed, let us formally decompose the observables as the sum of paths reaching the inclusion and paths that do no reach the inclusion:

$$
\begin{aligned}
\mathcal{E}^{\varepsilon} & =\sum_{\text {paths reaching } \Omega} e^{\varepsilon}+\sum_{\text {paths not reaching } \Omega} e^{\varepsilon}, \\
\mathcal{E}_{\text {inc }}^{\varepsilon} & =\sum_{\text {paths reaching } \Omega} e_{\text {inc }}^{\varepsilon}+\sum_{\text {paths not reaching } \Omega} e_{\text {inc }}^{\varepsilon}, \\
\mathcal{C}^{\varepsilon} & =\sum_{\text {paths reaching } \Omega} c^{\varepsilon}+\sum_{\text {paths not reaching } \Omega} c^{\varepsilon},
\end{aligned}
$$

for some densities $e^{\varepsilon}, e_{\mathrm{inc}}^{\varepsilon}$ and $c^{\varepsilon}$. Concerning the paths not reaching the inclusion, we have $e^{\varepsilon}=e_{\text {inc }}^{\varepsilon}=c^{\varepsilon}$, so that

$$
\mathcal{E}_{\text {inc }}^{\varepsilon}-\mathcal{E}^{\varepsilon}=\sum_{\text {paths reaching } \Omega}\left(e_{\text {inc }}^{\varepsilon}-e^{\varepsilon}\right), \quad \mathcal{C}^{\varepsilon}-\mathcal{E}^{\varepsilon}=\sum_{\text {paths reaching } \Omega}\left(c^{\varepsilon}-e^{\varepsilon}\right) .
$$

In the transport regime (i.e., when the mean free path is comparable to the overall distance over which propagation is observed), it is shown in [9] that the terms above are proportional to the surface of the inclusion. For instance, when the inclusion is a ball of radius $R$, then

$$
\mathcal{E}_{\mathrm{inc}}^{\varepsilon}-\mathcal{E}^{\varepsilon} \approx \mathcal{O}\left(R^{d-1}\right), \quad \mathcal{C}^{\varepsilon}-\mathcal{E}^{\varepsilon} \approx \mathcal{O}\left(R^{d-1}\right)
$$

In the diffusive regime, when the mean free path $l$ is small compared a typical distance of propagation, then we obtain [9] when $d \geq 3$, that

$$
\mathcal{E}_{\text {inc }}^{\varepsilon}-\mathcal{E}^{\varepsilon} \approx \mathcal{O}\left(R^{d}\right), \quad \mathcal{C}^{\varepsilon}-\mathcal{E}^{\varepsilon} \approx \mathcal{O}\left(R^{d-2}\right)
$$

In two dimensions the orders instead become $\mathcal{O}(R)$ and $\mathcal{O}\left(|\log R|^{-1}\right)$, respectively. This shows that in the diffusive regime, the effect of the inclusion on the measurements is stronger in the case of correlations than in the case of energies.

In the transport regime, even though the corrections on the correlation and on the energy are of the same order with respect to the size of the inclusion as mentioned above, there is still an interest in using correlations instead of energies provided the former can 
be measured. This is related to statistical stability considerations. Indeed, we know that the correlation vanishes at the location of the inclusion, so that $c^{\varepsilon}=0$ once the inclusion has been reached by some paths. Denoting by $\mathbb{E}\left\{e^{\varepsilon}\right\}$ the ensemble average of $e^{\varepsilon}$ and by $\delta e^{\varepsilon}:=e^{\varepsilon}-\mathbb{E}\left\{e^{\varepsilon}\right\}$ the instabilities, we thus have

$$
\begin{aligned}
\mathcal{C}^{\varepsilon}-\mathcal{E}^{\varepsilon} & =-\sum_{\text {paths reaching } \Omega} e^{\varepsilon}=-\sum_{\text {paths reaching } \Omega}\left(\mathbb{E}\left\{e^{\varepsilon}\right\}+\delta e^{\varepsilon}\right), \\
& =\mathbb{E}\left\{\mathcal{C}^{\varepsilon}-\mathcal{E}^{\varepsilon}\right\}-\sum_{\text {paths reaching } \Omega} \delta e^{\varepsilon}:=\mathbb{E}\left\{\mathcal{C}^{\varepsilon}-\mathcal{E}^{\varepsilon}\right\}+\delta C^{\varepsilon} .
\end{aligned}
$$

The measurements are $\mathcal{C}^{\varepsilon}-\mathcal{E}^{\varepsilon}$ and are approximated by the transport model for $\mathbb{E}\left\{\mathcal{C}^{\varepsilon}-\mathcal{E}^{\varepsilon}\right\}$ in the reconstructions. The random term $\delta C^{\varepsilon}$ is the source of noise in the reconstructions when using correlations. When using energy measurements instead, the source of noise is roughly twice as large. Indeed, in this case, the energy reaching the inclusion is not absorbed but rather reflected back so that we have

$$
\begin{aligned}
\mathcal{E}_{\mathrm{inc}}^{\varepsilon}-\mathcal{E}^{\varepsilon} & =\sum_{\text {paths reaching } \Omega}\left(e_{\mathrm{inc}}^{\varepsilon}-e^{\varepsilon}\right)=\sum_{\text {paths reaching } \Omega}\left(\mathbb{E}\left\{e_{\mathrm{inc}}^{\varepsilon}-e^{\varepsilon}\right\}+\delta e^{\varepsilon}-\delta e_{\mathrm{inc}}^{\varepsilon}\right), \\
& :=\mathbb{E}\left\{\mathcal{E}_{\mathrm{inc}}^{\varepsilon}-\mathcal{E}^{\varepsilon}\right\}+\sum_{\text {paths reaching } \Omega}\left(\delta e^{\varepsilon}-\delta e_{\mathrm{inc}}^{\varepsilon}\right):=\mathbb{E}\left\{\mathcal{E}_{\mathrm{inc}}^{\varepsilon}-\mathcal{E}^{\varepsilon}\right\}+\delta E^{\varepsilon} .
\end{aligned}
$$

Assuming that $\delta e^{\varepsilon}$ and $\delta e_{\text {inc }}^{\varepsilon}$ are comparable, we thus obtain $\delta E^{\varepsilon} \approx 2 \delta C^{\varepsilon}$. The noise caused by the statistical instabilities is approximately twice stronger for energies than for correlations. This fact can be seen on the reconstructions in section 4 . Correlations thus provide more stable results than energies even in the transport regime. They should thus be used when they can be measured.

For computational purposes, we consider the setting where measurements are available both in the presence and the absence of the inclusion since it requires smaller computational domains. We also assume that measurements can be performed at the wavelength scale so that we have access to the wave fields $\mathbf{u}_{1}^{\varepsilon}$ and $\mathbf{u}_{2}^{\varepsilon}$ and the correlation $\mathcal{C}^{\varepsilon}$ can be computed. Consider therefore that we know from wave measurements the quantities $\mathcal{E}_{\text {inc }}^{\varepsilon}-\mathcal{E}^{\varepsilon}$ and $\mathcal{C}^{\varepsilon}-\mathcal{E}^{\varepsilon}$. Statistical stability holds for spatially averaged quantities, so that we introduce for a given domain $\mathcal{D} \subset \mathbb{R}^{d}$ :

$$
\mathcal{E}^{\varepsilon}(t)=\int_{\mathcal{D}} \mathcal{E}^{\varepsilon}(t, \mathbf{x}) d \mathbf{x}, \quad \mathcal{E}_{\text {inc }}^{\varepsilon}(t)=\int_{\mathcal{D}} \mathcal{E}_{\text {inc }}^{\varepsilon}(t, \mathbf{x}) d \mathbf{x}, \quad \mathcal{C}^{\varepsilon}(t)=\int_{\mathcal{D}} \mathcal{C}^{\varepsilon}(t, \mathbf{x}) d \mathbf{x} .
$$

Following the statistical stability along with (7)-(8), we have

$$
\begin{aligned}
\mathcal{E}_{\text {inc }}^{\varepsilon}(t) & \approx \mathbb{E}\left\{\mathcal{E}_{\text {inc }}^{\varepsilon}\right\}(t) \approx \int_{\mathcal{D} \times \mathbb{R}^{d}} a_{2}^{+}(t, \mathbf{x}, \mathbf{k}) d \mathbf{x} d \mathbf{k} \\
\mathcal{E}^{\varepsilon}(t) & \approx \mathbb{E}\left\{\mathcal{E}^{\varepsilon}\right\}(t) \approx \int_{\mathcal{D} \times \mathbb{R}^{d}} a_{1}^{+}(t, \mathbf{x}, \mathbf{k}) d \mathbf{x} d \mathbf{k} \\
\mathcal{C}^{\varepsilon}(t) & \approx \mathbb{E}\left\{\mathcal{C}^{\varepsilon}\right\}(t) \approx \int_{\mathcal{D} \times \mathbb{R}^{d}} a_{12}^{+}(t, \mathbf{x}, \mathbf{k}) d \mathbf{x} d \mathbf{k} .
\end{aligned}
$$

The imaging procedure then consists of finding the transport predictions $a_{2}^{+}-a_{1}^{+}$and $a_{12}^{+}-a_{1}^{+}$, which depend on the inclusion, that best fit the measurements $\mathcal{E}_{\text {inc }}^{\varepsilon}-\mathcal{E}^{\varepsilon}$ 
and $\mathcal{C}^{\varepsilon}-\mathcal{E}^{\varepsilon}$. This requires in the first place that we estimate the power spectrum $\hat{R}$ and the mean free time $\Sigma^{-1}$ that characterize the transport equation (4). Once these parameters are known, an inverse transport problem is solved to find the inclusion location. Reconstructions will not be exact since $\int_{\mathcal{D} \times \mathbb{R}^{d}} a_{1}^{+}(t, \mathbf{x}, \mathbf{k}) d \mathbf{x} d \mathbf{k}$ approximates $\mathcal{E}^{\varepsilon}(t)$ (with the same conclusion holding for $\mathcal{E}_{\text {inc }}^{\varepsilon}$ and $\mathcal{C}^{\varepsilon}$ ) only in the limit $\varepsilon \rightarrow 0$ and will thus (weakly) depend on the realization of the random medium. We will quantify such instabilities numerically.

\section{Numerical results}

This section is devoted to the presentation of some numerical results. We first briefly recall the computational setting used for the resolution of the first-order system (3) and the radiative transport equation (4). The validity of transport equations for the energy and the correlation has been demonstrated for instance in $[8,9]$. We then estimate the transport parameters, namely the mean free time $\Sigma^{-1}$ in the chosen configuration since the cross section is constructed to be isotropic and present some simulations to verify the statistical stability of the reconstruction. Finally, we image an inclusion using the transport models in two settings: one configuration where the inclusion is hidden behind a blocker that prevents any coherent information at the detector, and one without blocker for inclusions of two different sizes in order to quantify the effects of the statistical instabilities as a function of the size of the object to be reconstructed.

\subsection{Computational setting}

We discretize the first-order hyperbolic system (3) for pressure $p(t, \mathbf{x})$ and velocity $\mathbf{v}(t, \mathbf{x})$ augmented with suitable initial conditions and surrounded by a perfectly matched layer $[12,13]$ using a standard finite element scheme of second order. In all our simulations, we assume that

$$
\rho_{0}=1, \quad \kappa^{\varepsilon}(\mathbf{x})=1+\sqrt{\varepsilon} \kappa_{1}\left(\frac{\mathbf{x}}{\varepsilon}\right),
$$

where $\kappa_{1}$ is a stationary mean-zero random variable. The average sound speed in thus $c_{0}=1$. The fluctuations are characterized by the two-point correlation function

$$
R(\mathbf{y})=\mathbb{E}\left\{\kappa_{1}(\mathbf{x}+\mathbf{y}) \kappa_{1}(\mathbf{x})\right\},
$$

and by their power spectrum $\hat{R}(\mathbf{k})$, the Fourier transform of $R$. The fluctuations of the compressibility $\kappa_{1}(\mathbf{y})$ have been carefully modeled to verify prescribed power spectra as in [8]. When we say that a medium has fluctuations of order $x \%$, we refer to the standard deviation of $\kappa_{1}$ (with respect to $\kappa_{0} \equiv 1$ ). The code is parallelized using the PETSc library, which allows for simulations on large domains. The initial condition is chosen so that only one frequency $|\mathbf{k}|$ is present at the transport level, at least approximately. More precisely, we choose:

$$
\mathbf{u}_{0}(\mathbf{x})=\left(\mathbf{0}, C_{0} \exp \left(\frac{-\left|\mathbf{x}-\mathbf{x}_{0}\right|^{2}}{2 \sigma^{2}}\right) J_{0}\left(\left|\mathbf{k}_{0}\right|\left|\mathbf{x}-\mathbf{x}_{0}\right|\right)\right)=\left(\mathbf{0}, p_{0}\right)
$$

where $J_{0}$ is the zero-th order Bessel function of the first kind. This initial condition exhibits an oscillatory behavior at the frequency $\mathbf{k} / \varepsilon$ (reduced frequency $\mathbf{k}$ ) and is 
localized in the vicinity of the point $\mathbf{x}_{0}$. The constant $C_{0}$ is chosen so that the energy associated to $\mathbf{u}_{0}$ is equal to one. The exponential term is chosen here to localize the source term. However, it has sufficiently slow variations in order not to interfere with the highly oscillatory Bessel function. Here, $\sigma$ is chosen to be on the order of ten wavelengths so that the frequency content of $\mathbf{u}_{0}$ is primarily that of a single wavenumber $\left|\mathbf{k}_{0}\right|$.

The transport equations are solved by a Monte Carlo method as in [8]. The modified transport equation on $a_{12}^{+}$is solved as usual, except that particles are killed when they hit $\partial \Omega$. The difference of intensities $a_{1}^{+}-a_{12}^{+}$is estimated by using the same variance reduction technique as in [8]: namely the random trajectories that do not hit $\partial \Omega$ are the same in the calculation of both $a_{+}^{1}$ and $a_{+}^{12}$. The initial condition at the transport level is

$$
a_{0}(\mathbf{x}, \mathbf{k})=\delta\left(\mathbf{x}-\mathbf{x}_{0}\right) \delta\left(|\mathbf{k}|-\left|\mathbf{k}_{0}\right|\right)\left|\mathbf{k}_{0}\right|^{-1} .
$$

The power spectrum $\hat{R}$ is chosen isotropic of the form

$$
\hat{R}(r)=\left\{\begin{array}{lcr}
\hat{R}_{0} & \text { for } & r<M, \\
0 & \text { for } & r>M
\end{array}\right.
$$

where $M$ is a given parameter such that $M>2\left|\mathbf{k}_{0}\right|$. The scattering coefficient $\Sigma$ is then given by

$$
\Sigma(k)=\frac{k^{3} \hat{R}_{0}}{4} .
$$

As explained earlier, we choose a relatively high scattering regime in which no coherent information is available. This corresponds to a random medium with $8 \%$ of standard deviation, namely $\sqrt{R_{0}}=8 \%$ with $R_{0}$ given by $(2 \pi)^{2} R_{0}=\pi M^{2} \hat{R}_{0}$. In this regime, we expect the correlations to offer more stable results than the energies as explained in section 3 .

Denoting by $\mathcal{D}$ the physical location of the array of detectors, we use the notations (9) for the wave description and introduce as well

$$
\mathcal{A}_{1}(t)=\int_{\mathcal{D}} \int_{\mathbb{R}^{2}} a_{1}^{+}(t, \mathbf{x}, \mathbf{k}) d \mathbf{x} d \mathbf{k}=\int_{\mathcal{D}} \int_{S^{1}} a_{1}^{+}\left(t, \mathbf{x}, \hat{\mathbf{k}}\left|\mathbf{k}_{0}\right|\right) 2 \pi\left|\mathbf{k}_{0}\right| d \mathbf{x} d \hat{\mathbf{k}}
$$

for the transport prediction. In the presence of an inclusion, the influence on the energies is denoted by $\delta \mathcal{E}_{\text {inc }}=\mathcal{E}^{\varepsilon}-\mathcal{E}_{\text {inc }}^{\varepsilon}$ for the wave fields and $\delta \mathcal{A}_{\text {inc }}=\mathcal{A}_{1}-\mathcal{A}_{2}$ for transport quantities, with

$$
\mathcal{A}_{2}(t)=\int_{\mathcal{D}} \int_{\mathbb{R}^{2}} a_{2}^{+}(t, \mathbf{x}, \mathbf{k}) d \mathbf{x} d \mathbf{k}=\int_{\mathcal{D}} \int_{S^{1}} a_{2}^{+}\left(t, \mathbf{x}, \hat{\mathbf{k}}\left|\mathbf{k}_{0}\right|\right) 2 \pi\left|\mathbf{k}_{0}\right| d \mathbf{x} d \hat{\mathbf{k}}
$$

The influence of the inclusion on the correlations is denoted by $\delta \mathcal{E}_{\text {cor }}=\mathcal{E}^{\varepsilon}-\mathcal{C}^{\varepsilon}$ for the wave fields and by $\delta \mathcal{A}_{\text {cor }}=\mathcal{A}_{1}-\mathcal{A}_{12}$ for the kinetic description, where

$$
\mathcal{A}^{12}(t)=\int_{\mathcal{D}} \int_{\mathbb{R}^{2}} a_{12}^{+}(t, \mathbf{x}, \mathbf{k}) d \mathbf{x} d \mathbf{k}=\int_{\mathcal{D}} \int_{S^{1}} a_{12}^{+}\left(t, \mathbf{x}, \hat{\mathbf{k}}\left|\mathbf{k}_{0}\right|\right) 2 \pi\left|\mathbf{k}_{0}\right| d \mathbf{x} d \hat{\mathbf{k}} .
$$

The energy is normalized so that $\mathcal{E}^{\varepsilon}(0)=\mathcal{E}_{\text {inc }}^{\varepsilon}(0)=\mathcal{C}^{\varepsilon}(0)=1$. 


\subsection{Estimation of the transport parameters}

We consider the following configuration: the domain of computation has the size $200 \lambda \times$ $200 \lambda$, with a wavelength $\lambda$ equal to one so that $\varepsilon=\frac{1}{200}=0.005$; the source emits a signal isotropically and is located at the point $(20,100)$. The detector is rectangular and delimited by the two extreme points $(10,70)$ and $(50,130)$. The configuration is depicted in figure 1. With our choice of the power spectrum $\hat{R}$, the cross section is isotropic, so that there is only one parameter to reconstruct, the mean free time $\Sigma^{-1}$. It is obtained by minimizing the quantity $\left\|\mathcal{E}^{\varepsilon}-\mathcal{A}_{1}[\Sigma]\right\|_{L^{2}\left(t_{0}, T\right)}\left\|\mathcal{E}^{\varepsilon}\right\|_{L^{2}\left(t_{0}, T\right)}^{-1}$ for 20 realizations of the random medium and provides the statistics of $\Sigma^{-1}$. We choose $T=1800$ and two different times $t_{0}, t_{0}=0$ and $t_{0}=270$. When $t_{0}=270$, the estimate of $\Sigma^{-1}$ will be more accurate for large times, times at which the effect of the inclusion can be seen. The minimization is done by brute force since there is only one parameter to find. For the case $t_{0}=0$, we find that the average of $\Sigma^{-1}$ is 40.80 with a standard deviation $\Delta \Sigma^{-1}=1.77$ (4.34\% of the average) and 40.25 with a standard deviation $1.33(3.30 \%$ of the average) when $t_{0}=270$. The estimate of $\Sigma^{-1}$ is slightly more stable (about $1 \%$ ) for $t_{0}=270$, which agrees with the fact that waves undergo more averaging at larger times. The theoretical value of $\Sigma^{-1}$ is 38.37. Accounting for the fact that numerical waves are slower than physical waves, see [8], so that the theoretical speed is 1 whereas the numerical speed is here 0.95 , we find a theoretical value for $\Sigma^{-1}$ of 40.39 , which is very close -by $1 \%$ or less- to the numerical values for both $t_{0}$. In the sequel, we set $\Sigma^{-1}=40.25$. In practical situations where we have access to only one realization, we expect the estimate of $\Sigma^{-1}$ to be in the range $\Sigma^{-1} \pm \Delta \Sigma^{-1}$ and no better.

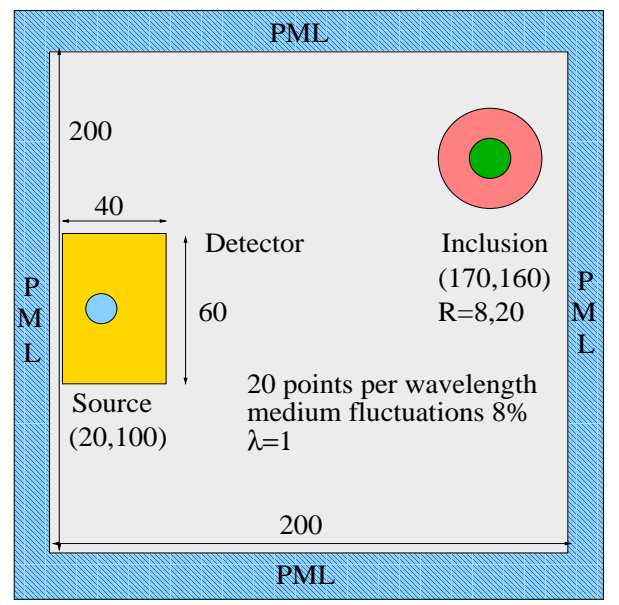

Figure 1: Domain of computation for the configurations without blocker. In configuration $1, R=20$ and in configuration $2, R=8$.

To quantity the error made when using $\Sigma^{-1} \pm \Delta \Sigma^{-1}$ instead of $\Sigma^{-1}$, we add a spherical inclusion with center $(X, Y)=(170,160)$ and radius $R=20$ and compare the resulting corrections $\delta \mathcal{A}_{\text {inc }}[\Sigma \pm \Delta \Sigma]$ and $\delta \mathcal{A}_{\text {inc }}[\Sigma]$ for the energies and $\delta \mathcal{A}_{\text {cor }}[\Sigma \pm \Delta \Sigma]$ and $\delta \mathcal{A}_{\text {cor }}[\Sigma]$ for the correlations. We find:

$$
\frac{\left\|\delta \mathcal{A}_{\text {inc }}[\Sigma \pm \Delta \Sigma]-\delta \mathcal{A}_{\text {inc }}[\Sigma]\right\|_{L^{2}(0, T)}}{\left\|\delta \mathcal{A}_{\text {inc }}[\Sigma]\right\|_{L^{2}(0, T)}} \approx \frac{\left\|\delta \mathcal{A}_{\mathrm{cor}}[\Sigma \pm \Delta \Sigma]-\delta \mathcal{A}_{\mathrm{cor}}[\Sigma]\right\|_{L^{2}(0, T)}}{\left\|\delta \mathcal{A}_{\mathrm{cor}}[\Sigma]\right\|_{L^{2}(0, T)}} \approx 6 \%,
$$


so that when using the average value of $\Sigma^{-1}$ to compute $\delta \mathcal{A}_{\text {inc }}[\Sigma]$ or $\delta \mathcal{A}_{\text {cor }}[\Sigma]$, we implicitly commit an error of about $6 \%$. We find similar errors for an inclusion of radius $R=8$. This means that if the method of reconstruction does not amplify errors and if we use a mean free time estimated from only one realization only -which we expect to be in the range $\left[\Sigma^{-1}-\Delta \Sigma^{-1}, \Sigma^{-1}+\Delta \Sigma^{-1}\right]$-, the results of reconstruction will be significant up to a standard deviation of about $6 \%$ in our configuration.

\subsection{Statistical stability}

As was done in $[8,9]$, we pursue our analysis of the statistical stability of energy measurements. In [9], it is shown that the larger the detector, the better is the stability and we investigate here the influence of the size of the support of the initial condition. Within the Itô-Schrödinger regime [1, 26], or for the single scattering scintillation of random Schrödinger equations with time-independent potentials, it is proved in [10] and [5] that waves emitted with an initial condition with a large support show a better stability than waves with a smaller support. We verify this property on the configuration depicted in figure 2 for an initial condition of the form (11) where $\sigma$ takes four different values: $\sigma=3,6,9,12$. The detector is located at the center of the domain and has a fairly large size so that we can assume that the instability is mainly related to the initial condition and not to the size of domain over which the energy is measured. We compute the following observable:

$$
S(t)=\frac{\left(\mathbb{E}\left[\left(\mathcal{E}^{\varepsilon}-\mathbb{E}\left[\mathcal{E}^{\varepsilon}\right]\right)^{2}\right](t)\right)^{1 / 2}}{\mathbb{E}\left[\mathcal{E}^{\varepsilon}\right](t)},
$$

where $\mathbb{E}$ denotes the average (mathematical expectation) computed over 20 realizations of the random medium and $\mathcal{E}^{\varepsilon}$ the energy averaged over the array of detectors. $S$ is a relative standard deviation. The results of the computation are depicted in figure 2 (right plot). For times larger than approximately 350, we observe what is predicted by the theory, namely that stability increases with the size of the support. For shorter times, this is not the case, and a possible explanation is that not enough averaging has occurred for such times.

As another illustration of the statistical instabilities, we represent in figure 3 the corrections $\delta \mathcal{E}_{\text {inc }}^{i}$ on the energy and $\delta \mathcal{E}_{\text {cor }}^{i}$ on the correlation for three different realizations of the random medium, $i=1,2,3$. The inclusion is located at $(170,160)$ and its radius is $R=8$, as in figure 1 . It can be clearly noticed that $\delta \mathcal{E}_{\text {cor }}^{i}$ is more stable than $\delta \mathcal{E}_{\text {inc }}^{i}$ as expected with the correlations, see section 3. For a larger inclusion with $R=20$, both energy and correlation measurements become more stable and have comparable stability properties since the correction due to the inclusion is larger and is therefore less sensitive to the random fluctuations.

\subsection{Configuration without blocker}

We reconstruct the inclusion in two different settings: in the first one, the inclusion is a ball located at $(X, Y)=(170,160)$, with radius $R=20$, and in the second one we set $R=8$ with same location. The inclusion is shifted in the cross-range direction to avoid a symmetrical situation. The configurations are represented in figure 1. 

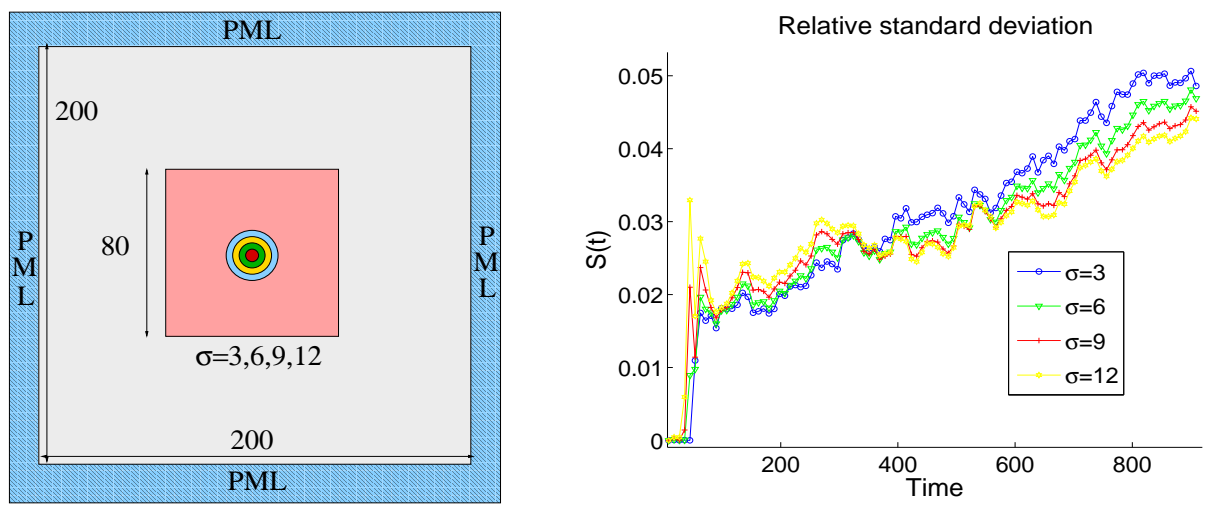

Figure 2: Left: domain of computation to evaluate the statistical stability according to the size of the support of the initial condition. The initial condition is a zero-th order Bessel function of the first kind localized by a Gaussian function with standard deviation $\sigma=3,6,9,12$. Right: relative standard deviation of the energy computed over 20 realizations for the four different sizes of the support.
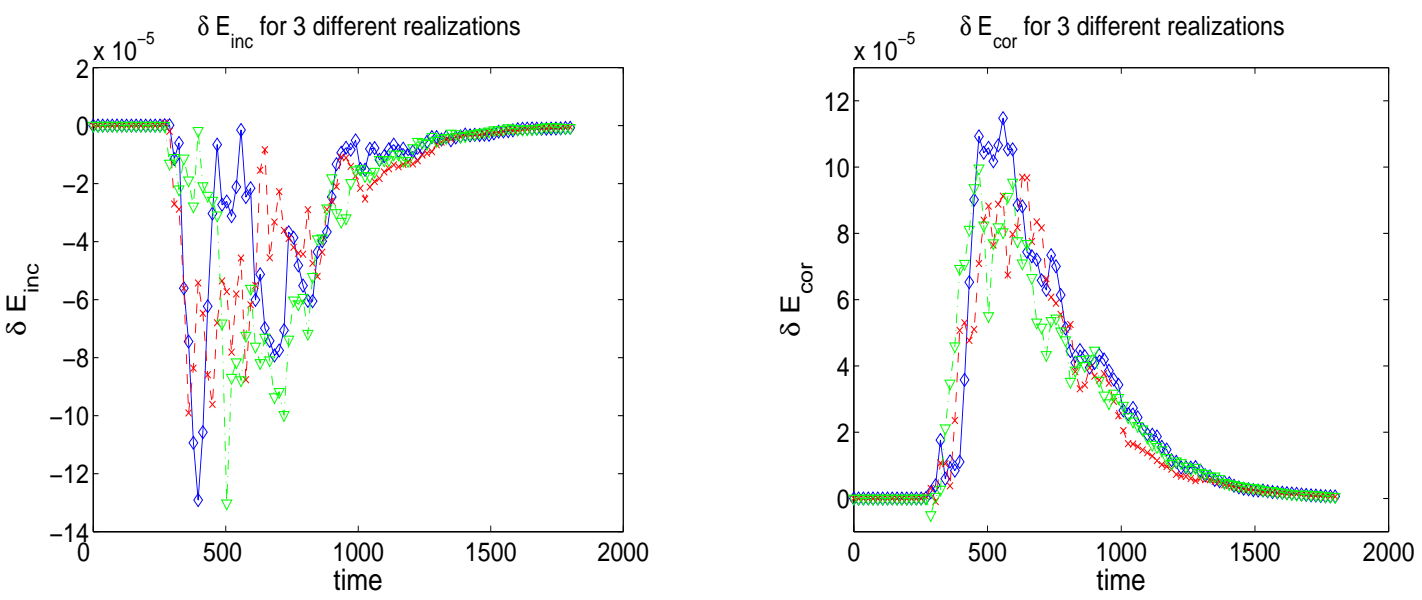

Figure 3: Representation of $\delta \mathcal{E}_{\text {inc }}$ and $\delta \mathcal{E}_{\text {cor }}$ for three different realizations of the random medium and a ball of radius 8 .

The simulations are performed for $N=20$ different realizations of the random medium and yield the set of measurements $\left(\mathcal{E}^{i}(t)\right)_{i=1, \ldots, N},\left(\mathcal{E}_{\text {inc }}^{i}(t)\right)_{i=1, \ldots, N}$ and $\left(\mathcal{C}^{i}(t)\right)_{i=1, \ldots, N}$ (we drop the $\varepsilon$ upper script) over the time interval $t \in[0, T]$. We thus have access to differential measurements. In addition to these data, we average the statistical instabilities out by computing the averages $\overline{\delta \mathcal{E}_{\text {inc }}}(t)$ and $\overline{\delta \mathcal{E}_{\text {cor }}}(t)$, defined as

$$
\overline{\delta \mathcal{E}_{\text {inc }}}(t)=\frac{1}{N} \sum_{i=1}^{N} \delta \mathcal{E}_{\text {inc }}^{i}(t), \quad \overline{\delta \mathcal{E}_{\text {cor }}}(t)=\frac{1}{N} \sum_{i=1}^{N} \delta \mathcal{E}_{\text {cor }}^{i}(t) .
$$

Such quantities would not be available in practical experiments since we would have access to one measurement only. Nevertheless, the corresponding reconstructed parameters are good indicators of the different errors in the model (finite value of $\varepsilon$, numerical scheme, etc...), excluding the error due to the statistical instabilities if we assume mean values computed over 20 realizations are accurate approximations of the true average. 
Since the data are quite irregular because of the statistical instabilities, we apply a Gaussian filter $G$ to smooth them out before solving the inverse problem. The effect of the filter is shown in Fig.4 for one realization and for an inclusion of radius $R=20$. To estimate the set of parameters $\mathcal{S}=(X, Y, R) \subset \mathbb{R}_{+}^{3}$, we minimize for $i=1, \cdots, 20$ realizations the quantities $\left\|G * \delta \mathcal{E}_{\text {inc }}^{i}-\delta \mathcal{A}_{\text {inc }}[\mathcal{S}]\right\|_{L^{2}(0, T)}\left\|\mathcal{E}^{i}\right\|_{L^{2}(0, T)}^{-1}$ for the energy and $\left\|G * \delta \mathcal{E}_{\text {cor }}^{i}-\delta \mathcal{A}_{\text {cor }}[\mathcal{S}]\right\|_{L^{2}(0, T)}\left\|\mathcal{E}^{i}\right\|_{L^{2}(0, T)}^{-1}$ for the correlation. $G * \delta \mathcal{E}_{\text {inc }}^{i}$ and $G * \delta \mathcal{E}_{\text {cor }}^{i}$ are the regularized versions of $\mathcal{E}_{\text {inc }}^{i}$ and $\mathcal{E}_{\text {cor }}^{i}$. We then obtain two distributions of the estimated parameters $\hat{S}_{\text {inc }}^{i}$ and $\hat{S}_{\text {cor }}^{i}, i=1, \cdots, 20$, which verify

$$
\begin{aligned}
& \hat{S}_{\mathrm{inc}}^{i}=\arg \min _{\mathcal{S} \subset \mathbb{R}_{+}^{3}} \frac{\left\|G * \delta \mathcal{E}_{\mathrm{inc}}^{i}-\delta \mathcal{A}_{\mathrm{inc}}[\mathcal{S}]\right\|_{L^{2}(0, T)}}{\left\|\mathcal{E}^{i}\right\|_{L^{2}(0, T)}}, \\
& \hat{S}_{\mathrm{cor}}^{i}=\arg \min _{\mathcal{S} \subset \mathbb{R}_{+}^{3}} \frac{\left\|G * \delta \mathcal{E}_{\mathrm{cor}}^{i}-\delta \mathcal{A}_{\mathrm{cor}}[\mathcal{S}]\right\|_{L^{2}(0, T)}}{\left\|\mathcal{E}^{i}\right\|_{L^{2}(0, T)}} .
\end{aligned}
$$
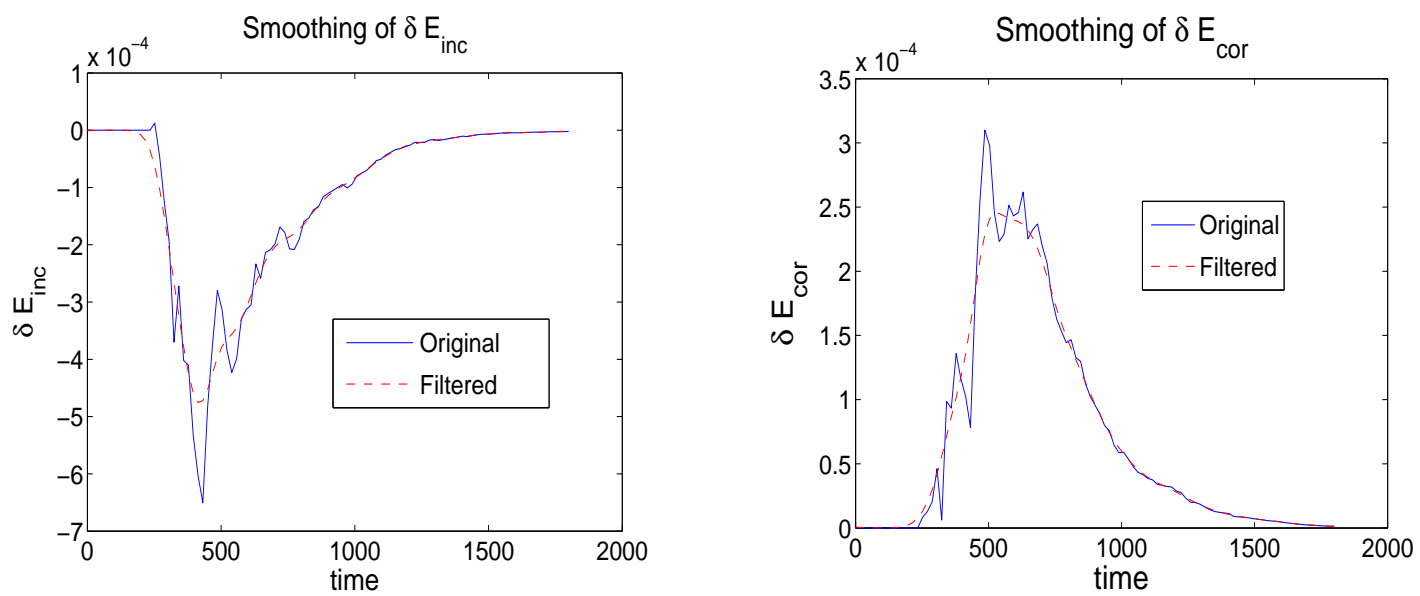

Figure 4: Regularization of $\delta \mathcal{E}_{\text {inc }}^{i}$ and $\delta \mathcal{E}_{\text {cor }}^{i}$ using a Gaussian filter.

We denote by $\overline{\mathcal{S}}_{\text {inc }}=\left(\bar{X}_{\text {inc }}, \bar{Y}_{\text {inc }}, \bar{R}_{\text {inc }}\right)$ the reconstructed parameters using $\overline{\delta \mathcal{E}_{\text {inc }}}$, and by $\hat{\mathcal{S}}_{\text {inc }}=\left(\hat{X}_{\text {inc }}, \hat{Y}_{\text {inc }}, \hat{R}_{\text {inc }}\right)$ and $\Delta \hat{\mathcal{S}}_{\text {inc }}=\left(\Delta \hat{X}_{\text {inc }}, \Delta \hat{Y}_{\text {inc }}, \Delta \hat{R}_{\text {inc }}\right)$ the mean value and standard deviation of $\left(\hat{S}_{\text {inc }}^{i}\right)_{i=1, \cdots, N}$. We use the same notation for the correlations.

The minimization procedure is quite simple since there are only three parameters to be reconstructed: we discretize the box $\left[X_{1}, X_{2}\right] \times\left[Y_{1}, Y_{2}\right] \times\left[R_{1}, R_{2}\right]$ and compute the value of $\left\|G * \delta \mathcal{E}_{\text {inc }}^{i}-\delta \mathcal{A}_{\text {inc }}[\mathcal{S}]\right\|_{L^{2}(0, T)}$ and $\left\|G * \delta \mathcal{E}_{\text {cor }}^{i}-\delta \mathcal{A}_{\text {cor }}[\mathcal{S}]\right\|_{L^{2}(0, T)}$ at each point of the grid to find the minimum.

We obtain the following values:

$$
\overline{\mathcal{S}}_{\text {inc }}=(168.26,161.65,19.63), \quad \overline{\mathcal{S}}_{\text {cor }}=(167.32,161.18,18.58),
$$

when $R=20$ and

$$
\overline{\mathcal{S}}_{\text {inc }}=(168.58,157.89,7.62), \quad \overline{\mathcal{S}}_{\text {cor }}=(169.84,157.41,7.47)
$$

when $R=8$. The results are plotted in Figs. 5 and 6 . We remark that in both configurations, the results are very close to the exact location of the inclusion, even 
though $\varepsilon$ is small but not zero $(\varepsilon=0.005)$ and the fact we used an elementary second order in time and space numerical scheme for the acoustic wave equation. Results are accurate by around 1\% for the position of the center using energies in configuration 1 and by $1.6 \%$ using correlations, while the errors on the radius are $2 \%$ and $7 \%$, respectively. For configuration 2, we find an error of less than $2 \%$ on the position for both energy and correlation measurements, while the error on the radius is around $6 \%$ for both observables. The larger error on the radius is likely due to the fact that radiative transfer is less precise close to the inclusion and that interference effects need to be accounted for.
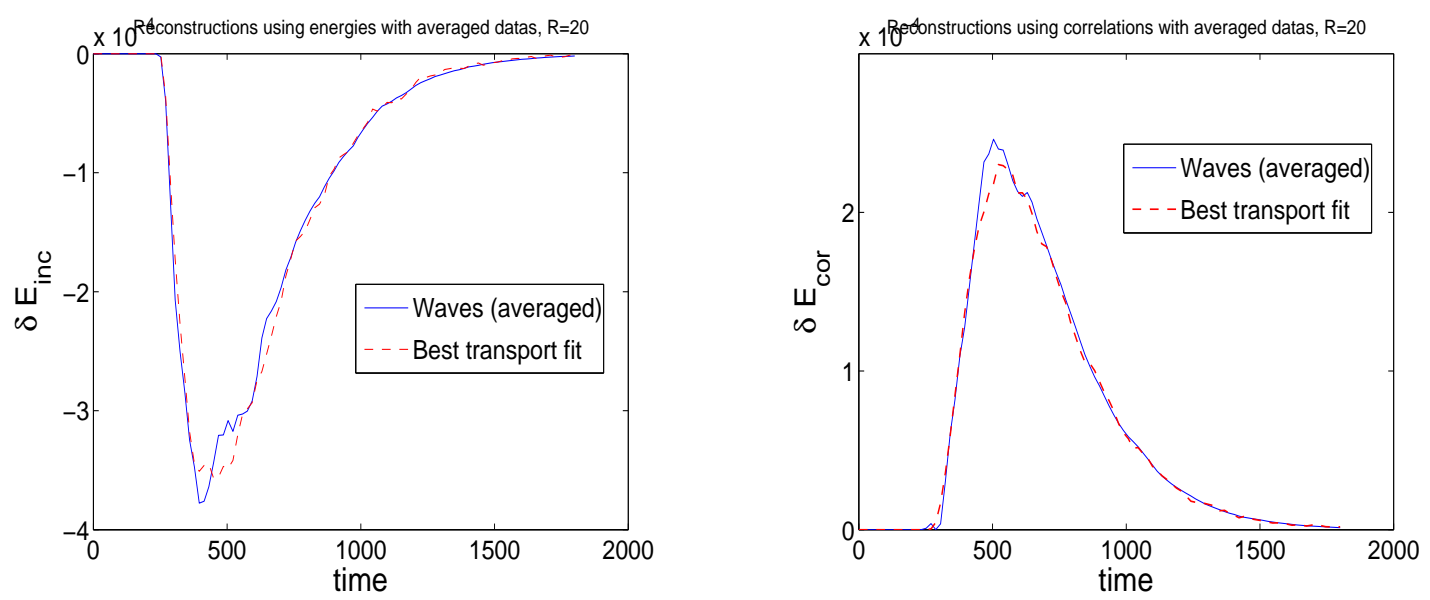

Figure 5: Reconstructions for configuration $1(\mathrm{R}=20)$ using averaged energies (left) and averaged correlations (right). The results are $\overline{\mathcal{S}}_{\text {inc }}=(168.26,161.65,19.63)$ and $\overline{\mathcal{S}}_{\text {cor }}=(167.32,161.18,18.58)$. The exact parameters are $(170,160,20)$.
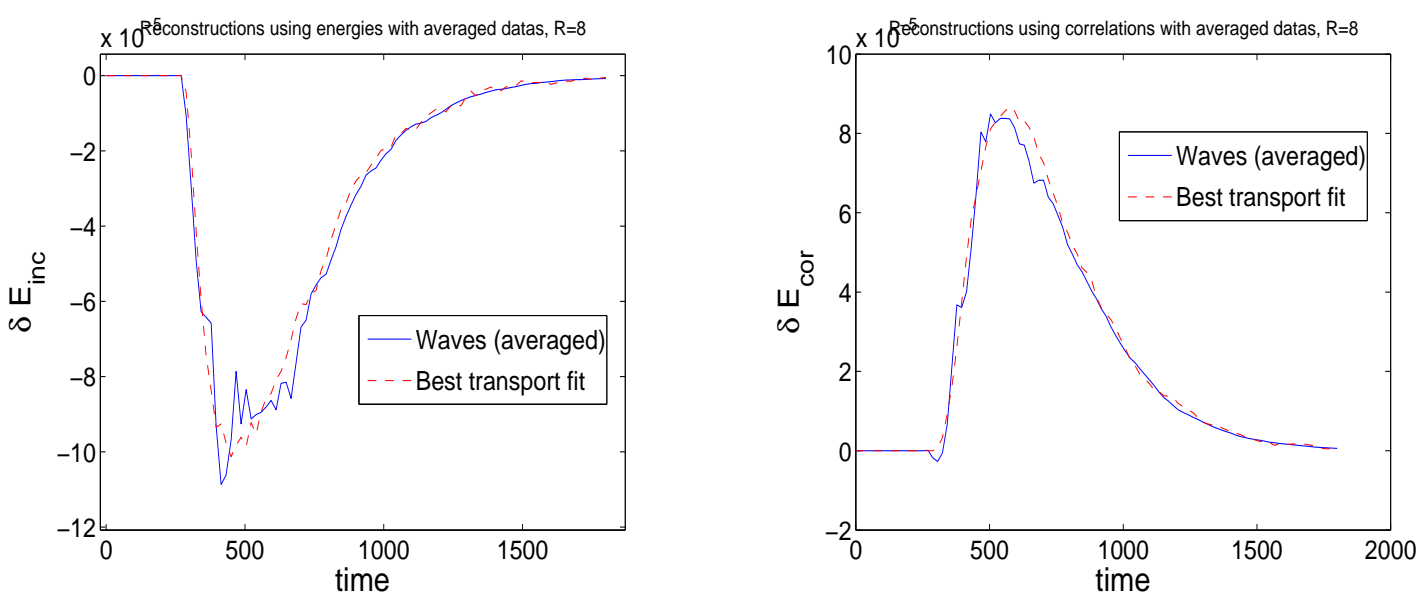

Figure 6: Reconstructions for configuration $2(\mathrm{R}=8)$ using averaged energies (left) and averaged correlations (right). The results are $\overline{\mathcal{S}}_{\text {inc }}=(168.58,157.89,7.62)$ and $\overline{\mathcal{S}}_{\text {cor }}=$ $(169.84,157.41,7.47)$. The exact parameters are $(170,160,8)$.

The results for the 20 different realizations are summarized in table 1 and displayed in Figs.7 and 8. For configuration 1, we find

$$
\hat{\mathcal{S}}_{\text {inc }}=(167.68,157.26,18.08), \quad \Delta \hat{\mathcal{S}}_{\text {inc }}=(4.30,3.04,2.11),
$$


for energies and

$$
\hat{\mathcal{S}}_{\text {cor }}=(169.26,158.25,18.7), \quad \Delta \hat{\mathcal{S}}_{\text {cor }}=(2.70,3.40,1.76) .
$$

\begin{tabular}{|c|c|c||c|c|}
\cline { 2 - 5 } \multicolumn{1}{c|}{} & \multicolumn{2}{c||}{ Configuration 1 $(R=20)$} & \multicolumn{2}{c|}{ Configuration 2 $(R=8)$} \\
\cline { 2 - 5 } \multicolumn{1}{c|}{} & energy & correlation & energy & correlation \\
\hline$R$ & 18.08 & 18.7 & 8.10 & 6.67 \\
\hline$\Delta R$ & 2.11 & 1.76 & 1.87 & 1.54 \\
\hline$X$ & 167.68 & 169.26 & 169.20 & 167.84 \\
\hline$\Delta X$ & 4.30 & 2.70 & 10.65 & 5.70 \\
\hline$Y$ & 157.26 & 158.25 & 162.64 & 156.45 \\
\hline$\Delta Y$ & 3.04 & 3.40 & 12.12 & 4.11 \\
\hline
\end{tabular}

Table 1: Results for the case without blocker, the exact location of the inclusion is $(X, Y)=(170,160)$ with radii $R=8$ or $R=20$. Statistics are performed over 20 realizations.

We remark that the results using energies and correlations are comparable. The average estimated position is very accurate with a standard deviation of around $2 \%$. The standard deviation for the radius is about 10\%. As expected, energies and correlations then provide comparable results since on the one hand the inclusion is large enough to yield a correction greatly above the statistical instabilities and on the other hand statistical instabilities have been smoothed out thanks to the regularization with the Gaussian filter. The situation is different for configuration 2 with a smaller inclusions of radius $R=8$. We find:

$$
\hat{\mathcal{S}}_{\text {inc }}=(169.20,162.64,8.10), \quad \Delta \hat{\mathcal{S}}_{\text {inc }}=(10.65,12.12,1.87),
$$

for energies and

$$
\hat{\mathcal{S}}_{\text {cor }}=(167.84,156.45,6.67), \quad \Delta \hat{\mathcal{S}}_{\text {cor }}=(5.70,4.11,1.54) .
$$

Reconstructions using correlations are a bit less accurate than with energies, $R=6.67$ to be compared with $R=8.10$ for instance, but are more stable. Indeed, we observe a standard deviation on the position of about $7 \%$ for energies with half this value for the correlations. Standard deviations are comparable for the radius. In this configuration, the statistical instabilities have more influence on the reconstructions since the correction due to the inclusion is weaker so that the signal to noise ration is lower than in the case $R=20$. As we have seen in section 3, noise is roughly twice bigger for energies than correlations, which explains the more stable reconstructions of the position of the center of the inclusion.

\subsection{Configuration with a blocker}

We now add a blocker between the source and the inclusion so that there is no coherent backscattered signal by the inclusion (no line of sight between the source and the inclusion). We suppose that we have access to differential measurements, which provide 

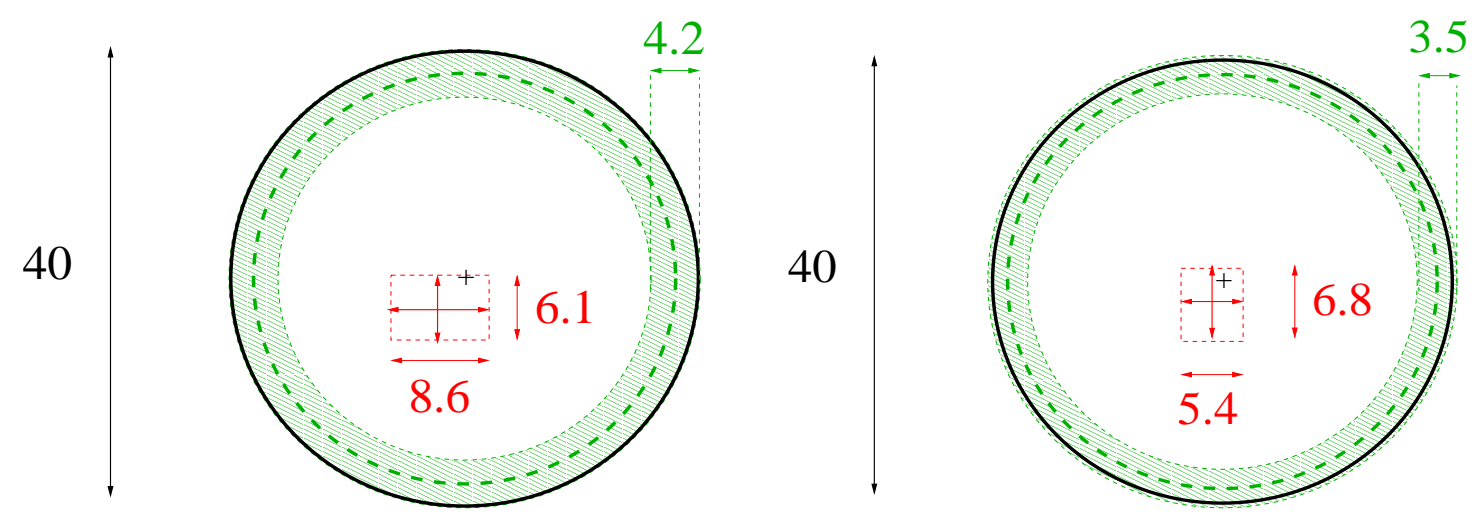

Figure 7: Reconstructions for configuration $1(\mathrm{R}=20)$ using energies (left) and correlations (right). The black circle represents the exact radius and the cross the exact location of the ball center. The dashed circle corresponds to the average reconstructed radius $\hat{R}$, and the shaded zone around circles of radii $\hat{R} \pm \Delta \hat{R}$. The center of the dashed rectangle is the average reconstructed ball center $(\hat{X}, \hat{Y})$ and its dimensions are $2 \Delta \hat{X} \times 2 \Delta \hat{Y}$.
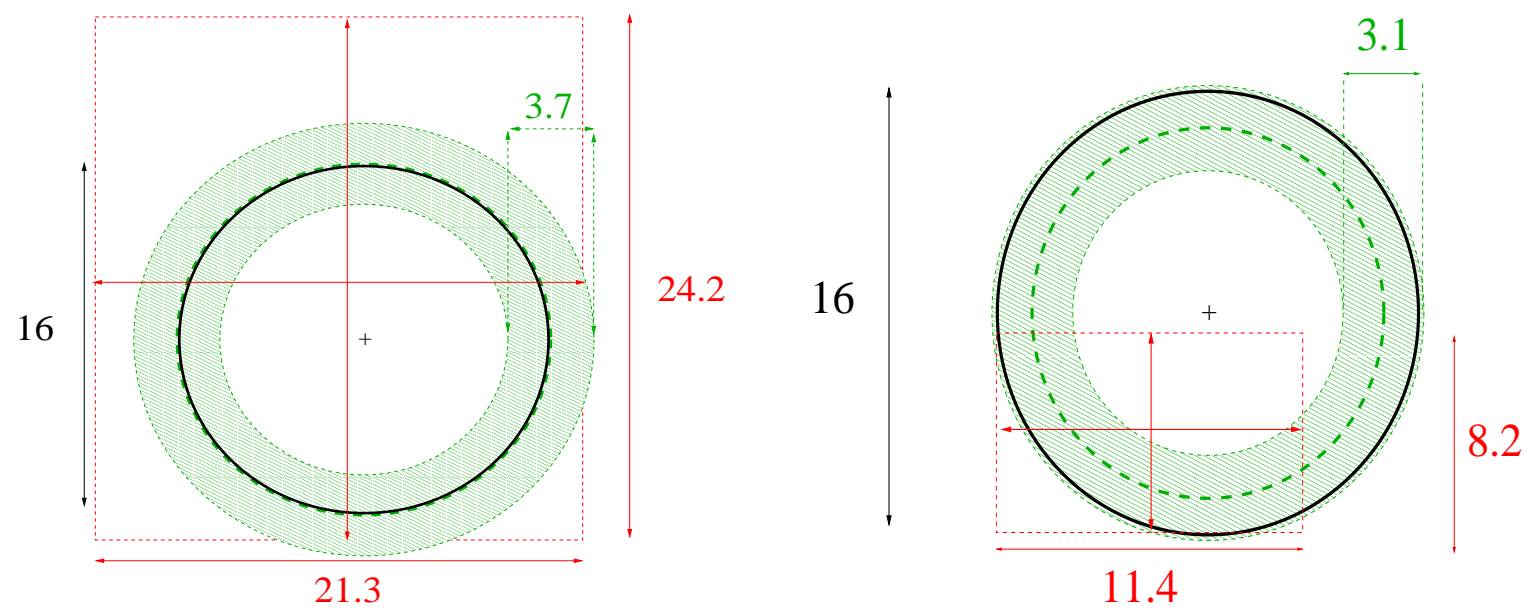

Figure 8: Reconstructions for configuration $2(\mathrm{R}=8)$ using energies (left) and correlations (right). The black circle represents the exact radius and the cross the exact location of the ball center. The dashed circle corresponds to the average reconstructed radius $\hat{R}$, and the shaded zone around circles of radii $\hat{R} \pm \Delta \hat{R}$. The center of the dashed rectangle is the average reconstructed ball center $(\hat{X}, \hat{Y})$ and its dimensions are $2 \Delta \hat{X} \times 2 \Delta \hat{Y}$.

the evolution of the energy averaged on the detector with and without the inclusion. For numerical considerations, the location of the blocker is supposed to be known. It is actually possible to reconstruct the location of the blocker and the inclusion at the same time provided the blocker is big enough to yield an influence larger than the statistical instabilities. Choosing such a large blocker will render the effect of inclusion very faint compared to the different errors in the models, statistical stability included, so that the resolution of the inverse problem becomes difficult. The computational domain is presented in Fig.9. We obtain the following values for reconstructions using the averaged data:

$$
\overline{\mathcal{S}}_{\text {inc }}=(172.68,156.20,18.53), \quad \overline{\mathcal{S}}_{\text {cor }}=(168.79,162.32,20.84) .
$$




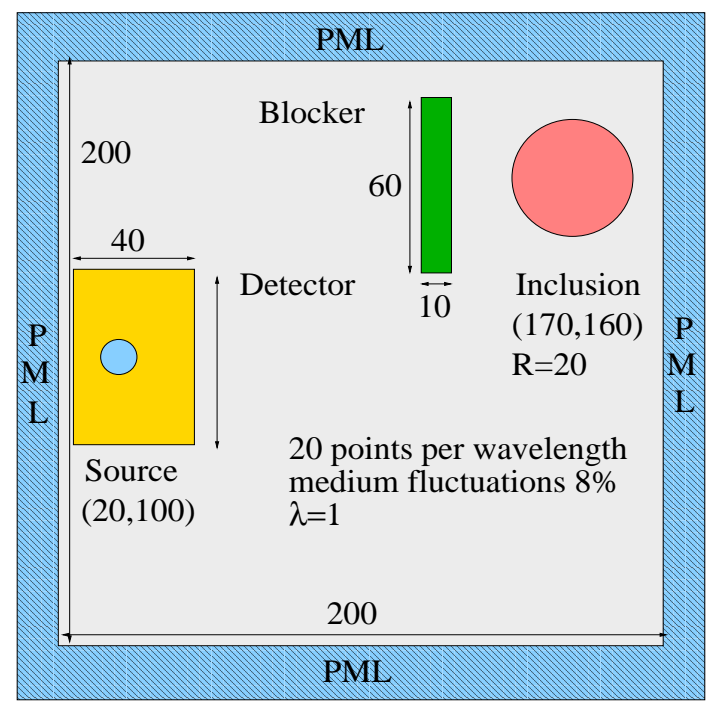

Figure 9: Domain of computation for a configuration with blocker for an inclusion of radius $R=20$.

Using the 20 different realizations of the random medium, we find with the energies:

$$
\hat{\mathcal{S}}_{\text {inc }}=(161.78,160.70,18.28), \quad \Delta \hat{\mathcal{S}}_{\text {inc }}=(17.76,11.97,2.44) .
$$

Using correlations, we have:

$$
\hat{\mathcal{S}}_{\text {cor }}=(160.46,161.58,19.49), \quad \Delta \hat{\mathcal{S}}_{\text {cor }}=(12.90,7.67,1.75) .
$$

The results are summarized in table 2 and presented in Fig.10. We remark that the average value of the radius is close to the exact value by about $9 \%$ and $2.5 \%$ using energies and correlations, respectively. The corresponding standard deviations are about $12.2 \%$ and $8.7 \%$. The average of the $X$ coordinates fits the exact value by about $6 \%$ for both energies and correlations, with standard deviations of $10 \%$ for the energy and $7.5 \%$ for the correlation. The average of the $Y$ coordinates fits the exact value by less than $1 \%$, with standard deviations of $7.5 \%$ for the energy and $4.8 \%$ for the correlation. Within this configuration, correlations yield a stability about $50 \%$ better than that of energies for the radius and the $Y$ coordinates. The increase in statistical instabilities is due to the presence of the blocker, which renders the measurements more faint and therefore more sensitive to the random fluctuations.

\section{Conclusion}

We have used radiative transfer equations to reconstruct an inclusion (a ball) embedded in a random medium with sufficiently strong disorder that methods based on wave propagation in a homogeneous medium are inaccurate. From wave measurements, the position and the radius of the ball were estimated by solving an inverse transport problem, the presence of an inclusion being seen as a perturbation of the transport parameters. Two different observables were considered as inputs for the inverse problem: the wave energy and the correlation between the wavefield in the presence of the inclusion and 

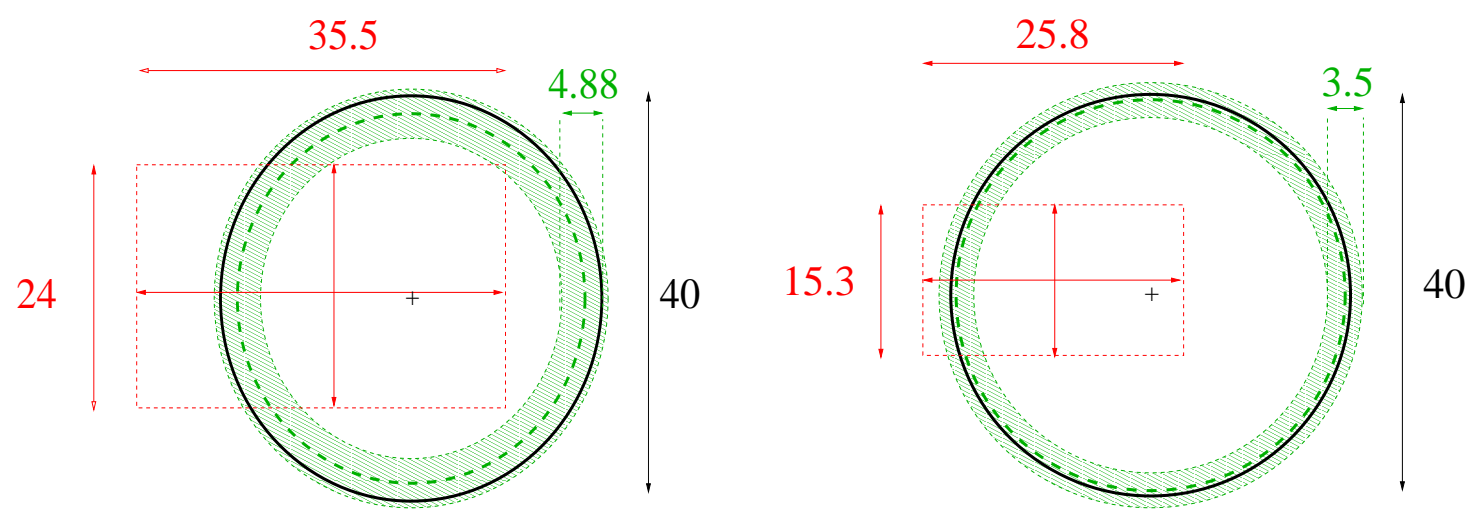

Figure 10: Reconstructions for configuration with blocker $(\mathrm{R}=20)$ using energies (left) and correlations (right). The black circle represents the exact radius and the cross the exact location of the ball center. The dashed circle corresponds to the average reconstructed radius $\hat{R}$, and the shaded zone around circles of radii $\hat{R} \pm \Delta \hat{R}$. The center of the dashed rectangle is the average reconstructed ball center and its dimensions are $2 \Delta \hat{X} \times 2 \Delta \hat{Y}$.

\begin{tabular}{|c|c|c|}
\cline { 2 - 3 } \multicolumn{1}{c|}{} & \multicolumn{2}{c|}{ Configuration with blocker } \\
\cline { 2 - 3 } \multicolumn{1}{c|}{} & energy & correlation \\
\hline$R$ & 18.28 & 19.49 \\
\hline$\Delta R$ & 2.44 & 1.75 \\
\hline$X$ & 161.78 & 160.46 \\
\hline$\Delta X$ & 17.76 & 12.90 \\
\hline$Y$ & 160.70 & 161.58 \\
\hline$\Delta Y$ & 11.97 & 7.67 \\
\hline
\end{tabular}

Table 2: Results for the case with a blocker. The exact location of the inclusion is $(X, Y)=(170,160)$ with radius $R=20$. The statistics are performed over 20 realizations.

X

the wavefield without an inclusion. We have seen that both quantities provide accurate results and that, for relatively small inclusions, correlation measurements yield more stable results than energy measurements. This is due to the different type of boundary conditions satisfied by the two observables at the inclusion's boundary: specular for the energy, Dirichlet for the correlation. Such reconstructions are possible since energy and correlation are statistically stable quantities in the limit of vanishing wavelength. Using the scattering properties of the random medium, we have also reconstructed inclusions hidden behind a blocker that prevents any coherent information from being measured at the array of detectors. 


\section{Acknowledgment}

GB acknowledges support from NSF Grant DMS-0804696. OP acknowledges support from the French ANR project QUATRAIN, quantum transport in nanostructures.

\section{References}

[1] G. BAL, On the self-averaging of wave energy in random media, SIAM Mult. Mod. Simul., 2(3) (2004), pp. 398-420.

[2] G. BAL, Kinetics of scalar wave fields in random media, Wave Motion, 43 (2005), pp. 132-157.

[3] _ Homogenization in random media and effective medium theory for high frequency waves, Discrete Contin. Dyn. Syst. Ser. B, 8 (2007), pp. 473-492 (electronic).

[4] G. Bal, T. Komorowski, and L. Ryzhik, Self-averaging of Wigner transforms in random media, Comm. Math. Phys., 242(1-2) (2003), pp. 81-135.

[5] G. Bal, I. Langmore, and O. Pinaud, Single scattering estimates for the scintillation function of waves in random media, Submitted, (2009).

[6] G. Bal, G. Papanicolaou, and L. Ryzhik, Self-averaging in time reversal for the parabolic wave equation, Stochastics and Dynamics, 4 (2002), pp. 507-531.

[7] G. Bal and O. Pinaud, Time reversal-based imaging in random media, Inverse Problems.

[8] — Accuracy of transport models for waves in random media, Wave Motion, 43(7) (2006), pp. 561-578.

[9] _ Kinetic models for imaging in random media, SIAM Multiscale Model. Simul., 6 (2007), pp. $792-819$.

[10] _ Self-averaging of kinetic models in random media, Kinetic and Related Models, 1(1) (2008), pp. 85-100.

[11] G. Bal And K. Ren, Transport-based imaging in random media, SIAM Applied Math., 68(6) (2008), pp. 1738-1762.

[12] J. P. Berenger, A perfectly matched layer for the absorption of electromagnetic waves, J. Comput. Phys., 114(2) (1994), pp. 185-200.

[13] — Three-dimensional perfectly matched layer for the absorption of electromagnetic waves, J. Comput. Phys., 127(2) (1996), pp. 363-379.

[14] L. Borcea, G. Papanicolaou, and C. Tsogka, Interferometric array imaging in clutter, Inverse Problems, 21 (2005), pp. 1419-1460.

[15] — Adaptive interferometric imaging in clutter and optimal illumination, Inverse Problems, 22 (2006), pp. 1405-1436.

[16] _ Coherent interferometry in finely layered random media, Multiscale Model. Simul., 5 (2006), pp. $62-83$ (electronic).

[17] — Optimal waveform design for array imaging, Inverse Problems, 23 (2007), pp. 1973-2020.

[18] S. Chandrasekhar, Radiative Transfer, Dover Publications, New York, 1960.

[19] J. F. Claerbout, Fundamentals of Geophysical Data Processing: With Applications to Petroleum Prospecting, Blackwell scientific, Palo Alto, 1985.

[20] M. Fink, Time reversed acoustics, Physics Today, 50(3) (1997), pp. 34-40.

[21] J.-P. Fouque, J. Garnier, G. Papanicolaou, and K. Sølna, Wave propagation and time reversal in randomly layered media, vol. 56 of Stochastic Modelling and Applied Probability, Springer, New York, 2007. 
[22] P. Gérard, P. A. Markowich, N. J. Mauser, and F. Poupaud, Homogenization limits and Wigner transforms, Comm. Pure Appl. Math., 50 (1997), pp. 323-380.

[23] A. Ishimaru, Wave Propagation and Scattering in Random Media, New York: Academics, 1978.

[24] P.-L. Lions and T. Paul, Sur les mesures de Wigner, Rev. Mat. Iberoamericana, 9 (1993), pp. 553-618.

[25] J. Lukkarinen and H. Spohn, Kinetic limit for wave propagation in a random medium, Arch. Ration. Mech. Anal., 183 (2007), pp. 93-162.

[26] G. Papanicolaou, L. Ryzhik, and K. Sølna, Self-averaging from lateral diversity in the ItôSchrödinger equation, Multiscale Model. Simul., 6 (2007), pp. 468-492 (electronic).

[27] L. Ryzhik, G. Papanicolaou, and J. B. Keller, Transport equations for elastic and other waves in random media, Wave Motion, 24 (1996), pp. 327-370.

[28] P. Sheng, Introduction to Wave Scattering, Localization and Mesoscopic Phenomena, Academic Press, New York, 1995.

[29] P. J. Shull, Nondestructive evaluation. Theory, Techniques and Applications, Marcel Dekker, New York, 2002. 